\title{
FINITE TYPE INVARIANTS OF INTEGRAL HOMOLOGY 3-SPHERES: A SURVEY
}

\author{
XIAO-SONG LIN \\ Department of Mathematics, University of California, Riverside, California 92521, U.S.A. \\ E-mail:xl@math.ucr.edu
}

1. Introduction. We are now embarrassingly rich in knot and 3-manifold invariants. We have to organize these invariants systematically and find out ways to make use of them. The theory of finite type knot invariants, or Vassiliev invariants, has been very successful in accomplishing the first task. Recently, an analogous theory of finite type invariants of integral homology 3 -spheres started to emerge. The analogy is mainly based on the common goal of bringing some order to the multitude of invariants by finding some universal properties they obey.

If we think of quantum 3-manifold invariants of Reshetikhin and Turaev [44] as nonperturbative ones, their perturbative version [38] is the other source of motivation for this developing theory of finite type invariants of integral homology 3 -spheres. Nonperturbative invariants are somehow packed together tightly so that they usually support some very rich algebraic structures. Perturbative invariants, on the other hand, seem to be quite independent with each other. One may see this from Theorem 3.1, the only new result in this paper, which claims that the space of finite type invariants of integral homology 3 -spheres is a polynomial algebra. This leads to the speculation that perturbative invariants may contain more geometrical or topological information than non-perturbative ones, at least to compensate the loss of algebraic richness. The recent study of Ohtsuki's perturbative invariants in [30] supports this speculation (see Theorem 8.1).

As the title says, this is a survey of the developing theory of finite type invariants of integral homology 3 -spheres. Of course, the way we present the material and the questions addressed depend on personal taste. But we do hope that we have sketched a more or less complete picture of the current status of this theory.

This paper is based on the lectures given in the workshop on knot theory at Banach International Center of Mathematics, Warsaw, July 1995. We would like to thank the

1991 Mathematics Subject Classification: Primary 57M.

Research supported in part by NSF.

The paper is in final form and no version of it will be published elsewhere. 
hospitality of Banach Center and local organizers. We also wish to thank Zhenghan Wang for continuing stimulating conversations, and to Stavros Garoufalidis and Jerry Levine for some comments.

2. Definitions and basic properties. Let $\lambda$ be an invariant of oriented $\mathbb{Z}$-homology 3 -spheres (Z्Z-HS's in short). We may define a difference operation on $\lambda$ with respect to a knot $K$ in a $\mathbb{Z}$-HS $M$ :

$$
\left(D^{ \pm} \lambda\right)(M, K)=\lambda(M)-\lambda\left(M_{K, \pm 1}\right)
$$

where $M_{K, \pm 1}$ is the $\mathbb{Z}$-HS obtained from $M$ by a \pm 1 -surgery on $K$. Roughly speaking, the invariant $\lambda$ is said to be of finite type if it vanishes under a finite iteration of the difference operation $D^{ \pm}$. See Definition 2.2 below. To be more specific, we proceed in a slightly different direction first.

An algebraically split link (ASL in short) in a $\mathbb{Z}$-HS $M$ is a link with unoriented and unordered components such that all its linking numbers are zero. Henceforth, all 3 -manifolds will be oriented $\mathbb{Z}$-homology 3 -spheres and all links will be \pm 1 -framed ASL's. We will denote by $\# L$ the number of components of a link $L$. If $L$ is a link in a $\mathbb{Z}$-HS $M$, we denote by $M_{L}$ the $\mathbb{Z}$-HS obtained from surgery on $L$. In particular, if $L$ is an empty link, then $M_{L}=M$.

Let $\mathcal{I}$ be the set of orientation preserving homeomorphism classes of $\mathbb{Z}$-HS's. Let $\mathcal{M}$ be the vector space over $\mathbb{Q}$ with $\mathcal{I}$ as a basis. For $M \in \mathcal{I}$ and a link $L$ in $M$, define an element $(M, L) \in \mathcal{M}$ by

$$
(M, L)=\sum_{L^{\prime} \subset L}(-1)^{\# L^{\prime}} M_{L^{\prime}}
$$

where $L^{\prime}$ runs through all sublinks (including the empty link) of $L$. Let $\mathcal{M}_{k}$ be the subspace of $\mathcal{M}$ spanned by $(M, L)$ for all $M$ and all $L$ with $\# L=k+1$. The space has a natural stratification:

$$
\mathcal{M}=\mathcal{M}_{-1} \supset \mathcal{M}_{0} \supset \cdots \supset \mathcal{M}_{k-1} \supset \mathcal{M}_{k} \supset \cdots
$$

For $A, B \in \mathcal{M}_{k-1}$, we denote $A \sim B$ if $A-B \in \mathcal{M}_{k}$. We have the following basic lemmas from [39].

Lemma 2.1. For any $(M, L) \in \mathcal{M}_{k-1}$, there is a link $J$ in $S^{3}$ with $\# J=k$ such that $(M, L) \sim\left(S^{3}, J\right)$.

Lemma 2.2. $\left(S^{3}, L\right) \sim\left(S^{3}, J\right)$ if $L$ and $J$ are surgery equivalent.

Lemma 2.3. Let $L$ and $J$ be the same link in $S^{3}$ with different \pm 1 framings. Let $s(L)$ be the product of framings of $L$. Then $s(L)\left(S^{3}, L\right) \sim s(J)\left(S^{3}, J\right)$.

We have to recall here the definition of surgery equivalence of links from [27].

Definition 2.1. A surgery modification on a link $L$ in $S^{3}$ is first to have a disk $B$ in $S^{3}$ intersecting $L$ only in its interior with zero algebraic intersection number. Then perform a \pm 1 surgery on $\partial B$ which will change $L$ to another link $J$ in $S^{3}$. Two links $L$ and $J$ are called surgery equivalent if one of them can be changed to another by a finite sequence of surgery modifications. 
From the lemmas above, we may think of $\mathcal{M}_{k-1} / \mathcal{M}_{k}$ as spanned by surgery equivalence classes of ASL's in $S^{3}$ with $k$ components, where an ASL $L$ in $S^{3}$ with $k$ components is regarded as an element $\left(S^{3}, L\right) \in \mathcal{M}_{k-1}$ with the understanding that the framings of $L$ are all +1 .

In [27], Levine showed that the surgery equivalence classes of ASL's with oriented and ordered components are classified by Milnor's triple $\mu$ - invariants. To be more specific, surgery equivalence classes of ASL's with $k$ oriented and ordered components are in oneone correspondence with collections of integers $\left\{\mu\left(i_{1} i_{2} i_{3}\right)\right\}$, where $i_{1}, i_{2}, i_{3}$ are distinct indices among $1, \ldots, k$, such that

$$
\left\{\begin{array}{l}
\mu\left(i_{1} i_{2} i_{3}\right)=\mu\left(i_{2} i_{3} i_{1}\right)=\mu\left(i_{3} i_{1} i_{2}\right) \\
\mu\left(i_{1} i_{2} i_{3}\right)=-\mu\left(i_{2} i_{1} i_{3}\right)
\end{array}\right.
$$

Such a collection of integers $\left\{\mu\left(i_{1} i_{2} i_{3}\right)\right\}$ is realized by an ASL with $k$ oriented and order components in the following way: First, for each unordered triple of indices $\left\{i_{1}, i_{2}, i_{3}\right\}$ with $\mu\left(i_{1} i_{2} i_{3}\right) \neq 0$, we construct an ASL with three oriented components named by $i_{1}, i_{2}, i_{3}$, respectively, such that its triple Milnor $\mu$-invariant is the given one $\mu\left(i_{1} i_{2} i_{3}\right)$. This can be done by using (some variations of) the Borromean rings. Then, by some appropriate band sums, we connect all circles with the same name together to get the desired ASL.

In [39], Ohtsuki showed that $\operatorname{dim}\left(\mathcal{M}_{k-1} / \mathcal{M}_{k}\right)<\infty$. The proof of this finiteness theorem from the point of view of Levine's classification of surgery equivalence classes of ASL's were given in [8] and [13]. We quote here the version in [13].

THeOREM 2.1. In $\mathcal{M}_{k-1} / \mathcal{M}_{k}$, each $A S L$ can be expressed as a linear combination of $A S L$ 's whose collections of triple $\mu$-invariants satisfy the following conditions:

1. each $\mu\left(i_{1} i_{2} i_{3}\right)$ is either 0 or 1 for certain fixed cyclic order of indices;

2. each index appears in at most two non-zero $\mu\left(i_{1} i_{2} i_{3}\right)$ 's.

Thus, we see that $\mathcal{M}_{k-1} / \mathcal{M}_{k}$ has a spanning set in one-one correspondence with graphs having $k$ edges such that

- each vertex is of valence either 1 or 3 ;

- each edge connects two distinct vertices;

- no three edges have the same set of end points;

- no edges are isolated.

Furthermore, it was proved in [8] that $\mathcal{M}_{k-1} / \mathcal{M}_{k}$ has a spanning set in one-one correspondence with graphs having $k$ edges such that

- each connected component is either a graph whose vertices are all of valence 3 or a graph with three edges and one valence 3 vertex, like the letter Y;

- each edge connects two distinct vertices;

- no three edges have the same set of end points.

Thus, we have the following theorem.

Theorem 2.2 (Garoufalidis-Levine). $\mathcal{M}_{3 n}=\mathcal{M}_{3 n+1}=\mathcal{M}_{3 n+2}$.

Now let us consider rational $\mathbb{Z}$-HS invariants. Such an invariant extends naturally to a linear functional on $\mathcal{M}$. 
Definition 2.2 (Ohtsuki [39]). A rational $\mathbb{Z}-H S$ invariant $\lambda$ is said to be a finite type invariant of order $\leq k$ if $\lambda \mid \mathcal{M}_{k}=0$.

Obviously, the set $\mathcal{O}_{k}$ of all finite type $\mathbb{Z}$-HS invariants of order $\leq k$ form a finite dimensional vector space over $\mathbb{Q}$. In fact, $\mathcal{O}_{k}$ is the dual of $\mathcal{M} / \mathcal{M}_{k}$ by definition. As a corollary of Theorem 2.2, we have the following theorem.

TheOREM 2.3 (Garoufalidis-Levine). $\mathcal{O}_{3 n}=\mathcal{O}_{3 n+1}=\mathcal{O}_{3 n+2}$.

In [7], a variation of Definition 2.2 was introduced. It is based on the notion of boundary links. A boundary link in a 3-manifold $M$ is a link whose components bound disjoint Seifert surfaces. Obviously, a boundary link is an ASL but not vice versa. Let $\mathcal{W}_{n}$ be the subspace of $\mathcal{M}$ spanned by pairs $(M, L)$ where $L$ is a boundary link in $M$ with $n+1$ components. A $\mathbb{Z}$-HS invariant $\lambda$ is said of order $\leq n$ in the sense of Garoufalidis if $\lambda \mid \mathcal{W}_{n}=0$. We denote by $\mathcal{G}_{n}$ the vector space of invariants of order $\leq n$ in the sense of Garoufalidis.

Theorem 2.4 (Garoufalidis-Levine [9]). $\mathcal{O}_{3 n} \subseteq \mathcal{G}_{n}$

It is conjectured in [9] that $\mathcal{G}_{n}=\mathcal{O}_{3 n}$.

Apparently, the space $\mathcal{G}_{n}$ is harder to work with than $\mathcal{O}_{3 n}$ and much less is known about it than about $\mathcal{O}_{3 n}$. But its relationship with Heegaard splittings of 3-manifolds might provide some additional useful information about finite type invariants and the topology of 3-manifolds.

3. The space of finite type invariants as a polynomial algebra. As observed in [7], if $\lambda \in \mathcal{O}_{k}$ and $\lambda^{\prime} \in \mathcal{O}_{l}$, then $\lambda \lambda^{\prime} \in \mathcal{O}_{k+l}$. Thus, the space of all finite type $\mathbb{Z}$-HS invariants is a graded commutative algebra. The purpose of this section is to show that the graded algebra of all finite type $\mathbb{Z}$-HS invariants is actually a graded commutative and cocommutative Hopf algebra. By the structure theorem of graded commutative and cocommutative Hopf algebras [34], we conclude that the graded algebra of all finite type $\mathbb{Z}$-HS invariants is a polynomial algebra generated by primitive invariants. Furthermore, our construction of the coproduct implies that primitive invariants are exactly those which are additive under the connected sum of 3-manifolds.

It might be of some interest to comment on the history of the proof, which we are going to present, of the fact that the graded algebra of finite type $\mathbb{Z}$-HS invariants is a graded commutative and cocommutative Hopf algebra. The same argument applied to finite type knot invariants was first presented by this author in an AMS regional conference and the West Coast Topology Symposium at Stanford University in the spring of 1992. Before that, it was known to Bar-Natan [2] that the graded algebra of weight systems on chord diagrams is a graded commutative and commutative Hopf algebra. Kontsevich's work [21], which also appeared in the spring of 1992, surpassed our direct argument by showing that the graded algebra of finite type knot invariants is isomorphic to the graded algebra of weight systems on chord diagram (but see the remark at the end of this section). Therefore, our direct argument that the graded algebra of finite type knot invariants is a graded commutative and cocommutative Hopf algebra never appeared in publication. 
In [11], the notion of weight systems for finite type invariants of $\mathbb{Z}$-homology 3 -spheres was introduced and it was shown that these weight systems form a graded commutative and cocommutative Hopf algebra. Since we don't know yet whether we could integrating every weight system in the sense of Garoufalidis and Ohtsuki to a $\mathbb{Z}$-HS invariant as in the case of knot invariants, it is worthwhile now to argue directly that the graded algebra of finite type $\mathbb{Z}$-HS invariants is also a graded commutative and cocommutative Hopf algebra. It is obvious from the construction that the map from finite type invariants to their weight systems is a graded Hopf algebra homomorphism .

To begin with, we denote by

$$
\mathcal{O}=\bigcup_{k=0}^{\infty} \mathcal{O}_{k}
$$

the space of all finite type $\mathbb{Z}$-HS invariants.

Lemma 3.1. For $\lambda \in \mathcal{O}_{k}$ and $\lambda^{\prime} \in \mathcal{O}_{l}$, let $\lambda \lambda^{\prime}$ be the multiplication of $\lambda$ and $\lambda^{\prime}$ as linear functionals on $\mathcal{M}$, we have $\lambda \lambda^{\prime} \in \mathcal{O}_{k+l}$.

Proof. If we think of $\lambda \mid \mathcal{M}_{n-1}$ as the $n$-th difference of $\lambda$ as in the case of knot invariants [3], this lemma is simply a corollary of the Leibniz formula for differences of the product of two functionals.

Let $\lambda$ be a $\mathbb{Z}$-HS invariant. We denote by $M \# N=N \# M$ the connected sum of two oriented 3-manifolds, which inherits an orientation from that of $M$ and $N$. Fix the factor $N$ in the connected sum and we will get a new $\mathbb{Z}$-HS invariant $\lambda_{N}$ :

$$
\lambda_{N}(M)=\lambda(M \# N) .
$$

LemMA 3.2. If $\lambda \in \mathcal{O}_{k}$, then $\lambda_{N} \in \mathcal{O}_{k}$.

Proof. This comes directly from the definition of $\mathcal{O}_{k}$.

We will chose a basis for $\mathcal{O}$ in the following way. Since

$$
\mathcal{O}_{0} \subset \mathcal{O}_{1} \subset \cdots \subset \mathcal{O}_{k-1} \subset \mathcal{O}_{k} \subset \cdots
$$

and each $\mathcal{O}_{k} / \mathcal{O}_{k-1}$ is finite dimensional, there is a basis $\left\{\omega_{0}, \omega_{1}, \ldots, \omega_{n}, \ldots\right\}$ of $\mathcal{O}$ such that for a certain sequence of non-negative integers $n_{0} \leq n_{1} \leq \cdots \leq k_{n} \leq \cdots,\left\{\omega_{0}, \omega_{1}, \ldots\right.$ $\left.\ldots, \omega_{n_{k}}\right\}$ is a basis for $\mathcal{O}_{k}$. Furthermore, we assume that $\omega_{0}=1$, i.e. the constant invariant assigning to every $\mathbb{Z}$-HS the number 1 , and $\omega_{n}\left(S^{3}\right)=0$ if $n>0$.

Now let $\lambda \in \mathcal{O}_{k}$, then $\lambda_{N} \in \mathcal{O}_{k}$. We write

$$
\lambda_{N}=\phi_{0}(N) \omega_{0}+\phi_{1}(N) \omega_{1}+\cdots+\phi_{n_{k}}(N) \omega_{n_{k}}
$$

where the coefficients $\phi_{0}(N), \phi_{1}(N), \ldots$, and $\phi_{n_{k}}(N)$ can be thought of as $\mathbb{Z}$-HS invariants.

LEMmA 3.3. The $\mathbb{Z}$-HS invariants $\phi_{0}, \phi_{1}, \ldots, \phi_{n_{k}}$ are of finite type. More precisely, if the order of $\omega_{l}$ is $\leq r$, then the order of $\phi_{l}$ is $\leq k-r$.

P r o of. The first conclusion is very easy to obtain: Simply notice that $\omega_{0}, \omega_{1}, \ldots, \omega_{n_{k}}$ are linearly independent and $\lambda_{N}(M)=\lambda(M \# N)$ by definition. Since $\lambda \in \mathcal{O}_{k}$, for every 
ASL $L$ in $N$ with $k+1$ components, we have

$$
\lambda_{(N, L)}(M)=\lambda(M \#(N, L))=0
$$

for all $M$. This implies that

$$
\phi_{0}((N, L))=\phi_{1}((N, L))=\cdots=\phi_{n_{k}}((N, L))=0 .
$$

The second conclusion is obtained using the same argument, but taking into consideration the way we chose the basis $\left\{\omega_{0}, \omega_{1}, \ldots, \omega_{n_{k}}\right\}$. We will consider links $J$ in $M$ and $L$ in $N$ such that $\# J+\# L=k+1$. We have

$$
\lambda_{(N, L)}((M, J))=\lambda((M \# N, J \coprod L))=0 .
$$

Then, we may argue as before to finish the proof.

We define a coproduct $\Delta: \mathcal{O} \rightarrow \mathcal{O} \otimes \mathcal{O}$ preserving the grading by

$$
\Delta(\lambda)=\omega_{0} \otimes \phi_{0}+\omega_{1} \otimes \phi_{1}+\cdots+\omega_{n_{k}} \otimes \phi_{n_{k}} .
$$

Lemma 3.4. The coproduct defined above is cocommutative. Moreover, if order $(\lambda)>0$, we have

$$
\Delta(\lambda)=1 \otimes \lambda+\lambda \otimes 1+\sum_{i, j} \alpha_{i} \otimes \beta_{j}
$$

with homogeneous elements $\alpha_{i}, \beta_{j}$ of positive orders such that $\operatorname{order}\left(\alpha_{i}\right)+\operatorname{order}\left(\beta_{j}\right)=$ $\operatorname{order}(\lambda)$

Proof. The first conclusion is because of the commutativity of the connected sum of 3-manifolds.

For the second conclusion, we first get $\phi_{0}=\lambda$ by taking $M=S^{3}$. So, we get the summand $1 \otimes \lambda$ in $\Delta(\lambda)$. The summand $\lambda \otimes 1$ then comes from the cocommutativity of $\Delta$.

A $\mathbb{Z}$-HS invariant $\lambda$ is called additive if

$$
\lambda(M \# N)=\lambda(M)+\lambda(N) .
$$

A $\mathbb{Z}$-HS invariant $\lambda \in \mathcal{O}$ is called primitive if

$$
\Delta(\lambda)=1 \otimes \lambda+\lambda \otimes 1
$$

LEMma 3.5. $\lambda \in \mathcal{O}$ is additive iff it is primitive.

Proof. This directly follows from the definition of the coproduct $\Delta$.

Now we may summarize the previous discussion into the following theorem.

THEOREM 3.1. $\mathcal{O}$ is a polynomial algebra generated by additive invariants.

Proof. By the previous lemmas, $\mathcal{O}$ is a graded commutative and cocommutative Hopf algebra. By the structure theorem of graded commutative and cocommutative Hopf algebras [34], we know $\mathcal{O}$ is a polynomial algebra generated by primitive invariants. The theorem follows since primitive invariants are exactly additive invariants.

Rem a r k. Instead of defining $\mathcal{M}$ as a vector space over a field (say $\mathbb{Q}$ ) spanned by all $\mathbb{Z}$-HS's, we may define it as the free $R$-module generated by all $\mathbb{Z}$-HS's, where $R$ is a commutative ring with unit 1 . The subspaces $\mathcal{M}_{k}$ then become submodules. As pointed 
out by Józef Przytycki, similar to the case of knots discussed in [40], the completion of $\mathcal{M}$ with respect to the stratification $\left\{\mathcal{M}_{k}\right\}$ is a commutative and cocommutative $R$-Hopf algebra. Again similar to the case of knots, the obvious question is whether the modules $\mathcal{M}_{k} / \mathcal{M}_{k+1}$ are torsion free.

4. Basic examples: Ohtsuki's invariants. We are mainly talking about generalities so far and haven't had any non-trivial finite type $\mathbb{Z}$-HS invariants yet. Our basic examples come from Ohtsuki's work [38]. In [38], Ohtsuki extracted a series of rational $\mathbb{Z}$-HS invariants from the $S U(2)$ quantum invariants of Reshetikhin and Turaev [44]. Physically, they correspond to the coefficients of the asymptotic expansion of Witten's Chern-Simons path integral at the trivial connection as shown by Rozansky [41, 42]. Rozansky has also argued, based on some physical considerations, that Ohtsuki's invariants are all of finite type.

Let first us explain briefly how Ohtsuki's invariants are derived.

Let $\tau_{r}(M)$ be the $S U(2)$ quantum invariant of Reshetikhin and Turaev at the $r$-th root of the unit $q=e^{2 \pi \sqrt{-1} / r}$, as normalized in [20]. From [37], we know that if $M$ is a $\mathbb{Z}$-HS, $\tau_{r}(M) \in \mathbb{Z}[q]$ when $r$ is an odd prime. So we may write

$$
\tau_{r}(M)=a_{r, 0}+a_{r, 1}(q-1)+\cdots+a_{r, n}(q-1)^{n}+\cdots
$$

for $a_{r, n} \in \mathbb{Z}$. We will fix $n$ and think of $a_{r, n}$ as a function of $r$. We ask whether there is a rational number $\lambda_{n}$, independent of $r$, such that

$$
a_{r, n} \equiv \lambda_{n} \quad \bmod r
$$

is true for all odd primes $r$ sufficiently large. Of course, since $q$ is not an indeterminant, $a_{r, n}$ here is not well defined. Nevertheless, as $q^{r}=1, a_{r, n}$ is well defined modulo $r$ and our question is thus well posed.

The situation here is rather like the one dealt with in the so-called Fermat's little theorem: Fix an integer $a, a^{r-1} \equiv 1 \bmod r$ for all odd primes $r$ sufficiently large. From elementary number theory [16], we know that things are not always so nice. For example, $\left(\frac{r-1}{2}\right) ! \bmod r$ depends on $r$. For this reason, we call in [30] the formal power series

$$
\sum_{n=0}^{\infty} \lambda_{n}(t-1)^{n}
$$

the Fermat limit of $\tau_{r}(M)$ if (4.1) is true for every $n$, and we denote

$$
\mathrm{f}-\lim \tau_{r}(M)=\sum_{n=0}^{\infty} \lambda_{n}(t-1)^{n} .
$$

Certainly, if the Fermat limit of $\tau_{r}(M)$ exists, it is unique.

TheOrem 4.1 (Ohtsuki [38], see also [30]). The Fermat limit of $\tau_{r}(M)$ exists. In particular, we get a sequence of rational $\mathbb{Z}$-HS invariants $\lambda_{n}=\lambda_{n}(M)$.

It is quite easy to get $\lambda_{0}=1$. If we denote by $\lambda_{C}$ the Casson invariant of $\mathbb{Z}$-HS's $[1]$, we have the following theorem.

Theorem 4.2 (Murakami [37]). $\lambda_{1}=6 \lambda_{C}$. 
The first example of non-trivial finite type $\mathbb{Z}$-HS invariants comes from $\lambda_{C}$, or equivalently, $\lambda_{1}$. We combine many known facts into the following theorem. See $[39,7]$.

THEOREM 4.3. We have

1. $\mathcal{O}_{0}=\mathcal{O}_{1}=\mathcal{O}_{2} \cong \mathbb{Q}$ and it is spanned by the constant invariant 1 ;

2. $\mathcal{O}_{3}=\mathcal{O}_{4}=\mathcal{O}_{5} \cong \mathbb{Q}^{2}$ and it is spanned by 1 and $\lambda_{C}$.

Following Theorem 2.3, we only need to explain why $\lambda_{C}$ is of order $\leq 3$. We proceed using the surgery formula of $\lambda_{C}$ given in [17].

Let $\nabla(L ; z)$ be the Conway polynomial [19] of a link $L$ in $S^{3}$. Then,

$$
\nabla(L ; z)=z^{\# L-1}\left(1+a_{2}(L) z^{2}+a_{4}(L) z^{4}+\cdots+a_{2 n}(L) z^{2 n}\right) .
$$

If $L$ be a \pm 1 -framed ASL, we will denote by $f_{L}$ the product of framings of $L$.

TheOrem 4.4 (Hoste [17]). Let $L$ be a \pm 1 -framed $A S L$ in $S^{3}$, then

$$
\lambda_{C}\left(S_{L}^{3}\right)=\sum_{L^{\prime} \subset L} f_{L^{\prime}} a_{2}\left(L^{\prime}\right)
$$

The following is a very interesting property of the link invariant $a_{2}$ on ASL's.

LEMma 4.1 (Hoste [17]). If $L$ is an $A S L$ with $\# L>3$, then $a_{2}(L)=0$.

We will leave it as an exercise for the reader to show that $\lambda_{C}$ is of order $\leq 3 \mathrm{using}$ Theorem 4.4 and Lemma 4.1. Since $\lambda_{C}$ is not a constant invariant, it is of order 3 .

It seems to be a common belief that $\lambda_{n}$ should be of order $3 n$ in general, and this has been verified physically by Rozansky $[41,42]$. Surgery formulae for $\lambda_{n}$ were given in [30]. Unfortunately, some coefficients in these formulae can only be determined recursively. We don't have close formulae yet to express these coefficients appeared in the surgery formulae for $\lambda_{n}$ when $n>2$. This makes it difficult to prove that $\lambda_{n}$ is of order $3 n$ for $n>3$. On the other hand, the explicit surgery formula for $\lambda_{2}$ in [30] makes it possible to prove that $\lambda_{2}$ is of order 6 .

Theorem 4.5 (Lin-Wang [31]). $\lambda_{2}$ is of order 6 .

We will briefly explain the proof of this theorem in the next section when the surgery formula for $\lambda_{2}$ is given.

In [11], it is estimated that $\operatorname{dim} \mathcal{O}_{6} \leq 4$ by using some relations among trivalent graphs representing $\mathbb{Z}$-HS's. On the other hand, a simple computation using the surgery formula for $\lambda_{2}$ (see (5.1) below) shows that $\lambda_{1}^{2}$ and $\lambda_{2}$ are linearly independent order 6 invariants. Thus,

$$
\mathcal{O}_{6}=\mathcal{O}_{7}=\mathcal{O}_{8} \cong \mathbb{Q}^{4}
$$

and it is spanned by $1, \lambda_{1}, \lambda_{1}^{2}, \lambda_{2}$.

5. The surgery formula for $\lambda_{2}$. We will denote by $O$ the unknot and $\emptyset$ the empty link.

The Jones polynomial $V(L ; t) \in \mathbb{Z}\left[t^{ \pm \frac{1}{2}}\right]$ of a link $L$ in $S^{3}$ is defined by

$$
\left\{\begin{array}{l}
V(O ; t)=1 \\
V(\emptyset ; t)=\left(t^{\frac{1}{2}}+t^{-\frac{1}{2}}\right)^{-1} \\
t V\left(L_{+} ; t\right)-t^{-1}\left(L_{-} ; t\right)=\left(t^{\frac{1}{2}}-t^{-\frac{1}{2}}\right) V\left(L_{0} ; t\right)
\end{array}\right.
$$


Here, as usual, $L_{+}, L_{-}$and $L_{0}$ are three links which have plane projections identical to each other except in one small disk where their projections are a positive crossing, a negative crossing and an orientation smoothing of that crossing, respectively. Note that the Jones polynomial we use here differs from the usual definition in [18] with a change of variable $t$ to $t^{-1}$ and a factor of $(-1)^{\# L-1}$.

We put

$$
X(L ; t)=\frac{V(L ; t)}{\left(t^{\frac{1}{2}}+t^{-\frac{1}{2}}\right) \# L-1}
$$

and

$$
\Phi(L, t)=\sum_{L^{\prime} \subset L}(-1)^{\# L-\# L^{\prime}} X\left(L^{\prime} ; t\right)
$$

where the sum runs through all sublinks of $L$. We need to use the derivatives of $\Phi(L ; t)$ at $t=1$ :

$$
\Phi_{i}(L)=\left.\frac{d^{i} \Phi(L ; t)}{d t^{i}}\right|_{t=1} .
$$

The basic link invariants used in formulae for $\lambda_{n}$ 's are

$$
\phi_{i}(L)=\frac{(-2)^{\# L}}{(\# L+i) !} \Phi_{\# L+i}(L)
$$

for $i=1,2, \ldots$ (see [30]). According to Murakami [37], we have

$$
\phi_{1}(L)=6 a_{2}(L)
$$

if $L$ is an ASL.

Given a link $L$ and a positive integer $m$, we denote by $L^{m}$ the 0 -framed $m$-parallel of $L$, i.e. each component in $L$ is replaced by $m$ parallel copies having linking number zero with each other. So $L^{m}$ is an ASL if $L$ is. Sublinks of $L^{m}$ will be assumed to be in one-one correspondence with \#L-tuples $\left(i_{1}, i_{2}, \ldots, i_{\# L}\right)$, in such a way that the corresponding sublink $L^{\prime}$ will have $i_{\xi}$ parallel copies of the $\xi$-th component of $L, 0 \leq i_{\xi} \leq m$. If $L$ is a framed link, $L^{m}$ and all its sublinks will inherit a framing from that of $L$.

THEOREM 5.1 (Lin-Wang [30]). Let L be a \pm 1 -framed ASL. Then

$$
\lambda_{2}\left(S_{L}^{3}\right)=\sum_{L^{\prime} \subset L} \phi_{1}\left(L^{\prime}\right) f_{L^{\prime}} \frac{\# L^{\prime}}{2}+\sum_{L^{\prime} \subset L^{2}} \phi_{2}\left(L^{\prime}\right) f_{L^{\prime}} \frac{1}{2^{s_{2}\left(L^{\prime}\right)}} .
$$

Here, if $L^{\prime} \subset L$ corresponds to the \#L-tuple $\left(i_{1}, i_{2}, \ldots, i_{\# L}\right), s_{2}\left(L^{\prime}\right)=\#\left\{i_{\xi} ; i_{\xi}=2\right\}$.

Similar to the proof that $\lambda_{C}$ is of order $\leq 3$, the following lemma together with Theorem 5.1 implies that $\lambda_{2}$ is of order $\leq 6$. A little more computation will then establish Theorem 4.5.

Lemma 5.1 (Lin-Wang [31]). If $L$ is an $A S L$ with $\# L>6$, then $\phi_{2}(L)=0$

The proof of this lemma is based on studying the colored Jones polynomial [44]. A generalization of (the first part of) the so-called Melvin-Morton conjecture [33, 5] for ASL's turns out to be crucial. 
6. Induced knot invariants. Let $\lambda$ be a $\mathbb{Z}$-HS invariant. It induces a knot invariant in the following way: For a knot $K$ in $S^{3}$, we assign to it the framing 1 . Then we define a knot invariant $\psi_{\lambda}$ by

$$
\psi_{\lambda}(K)=\lambda\left(S_{K}^{3}\right)
$$

LEMMA 6.1. If $\lambda$ is of finite type, so is $\psi_{\lambda}$ as a knot invariant.

This is quite obvious: A crossing change crossings can be accomplished by a \pm 1 -surgery on an appropriately positioned unknot. In fact, the proof of this lemma shows that if $\lambda$ is of order $\leq k$, then $\psi_{\lambda}$ is of order $\leq k-1$. The fact that the order of a finite type $\mathbb{Z}$-HS invariant must be a multiple of 3 (Theorem 2.3) somehow implies a stronger estimate on the order of the induced knot invariant.

Theorem 6.1 (Habegger [15], see also [9]). If $\lambda$ is a $\mathbb{Z}$-HS invariant of order $3 n, \psi_{\lambda}$ is a knot invariant of order $\leq 2 n$.

For example, the induced knot invariant of $\lambda_{C}$ is $a_{2}$, i.e. the second coefficient of the Conway polynomial. The induced knot invariant of $\lambda_{2}$ is slightly more complicated but can be obtained using (5.1). To express it using classical knot invariants, we denote

$$
v_{i}(L)=\left.\frac{d^{i} V\left(L ; e^{h}\right)}{d h^{i}}\right|_{h=0} .
$$

Let $\psi_{2}$ be the knot invariant induced by $\lambda_{2}$, we have

$$
\psi_{2}(K)=\frac{3}{2} v_{2}(K)-\frac{1}{3} v_{3}(K)+\frac{5}{3} v_{2}^{2}(K)-60 a_{4}(K) .
$$

7. More on Ohtsuki's invariants: their integrality. A priori, Ohtsuki's invariants $\lambda_{n}$ are rational invariants. From Theorem 4.2 , we see that $\lambda_{1} \in 6 \mathbb{Z}$. The following theorem follows from the surgery formula (5.1) for $\lambda_{2}$ and a careful study of the coefficients of the colored Jones polynomial of ASL's.

TheOREM 7.1 (Lin-Wang [30]). $\lambda_{2} \in 3 \mathbb{Z}$.

In [30], it is conjectured that $n ! \lambda_{n} \in 6 \mathbb{Z}$. This conjecture is certainly motivated by Theorems 4.2 and 7.1. But we do have some other evidence supporting this conjecture. For example, it is proved in [30] that the denominator of $\lambda_{n}$ can always be factored by some powers of $2,3, \ldots, n$.

8. Some applications of Ohtsuki's invariants. Formula (5.1) allows us to compute $\lambda_{2}$ quite easily. We may then use the invariant $\lambda_{2}$ to distinguish various $\mathbb{Z}$-HS's. For example, if $M$ is the Poincaré homology 3-sphere $\Sigma(2,3,5)(+1$-surgery on the righthanded trefoil knot), we have $\lambda_{2}(M)=39$. And if $M$ is the homology 3-sphere $\Sigma(2,3,7)$ $\left(+1\right.$-surgery on the left-handed trefoil knot), we have $\lambda_{2}(M)=63$. In general, we may use $\lambda_{2}$ to distinguish $\mathbb{Z}$-HS's obtained from $1 / n$-surgeries on a knot $K$ and its mirror image $K^{*}$, respectively, provided that $v_{3}(K) \neq 0$.

Theorem 8.1 (Lin-Wang [30]). If $v_{3}(K) \neq 0$, then $S_{K, 1 / n}^{3} \neq S_{K^{*}, 1 / n}^{3}$ for each $n \neq 0$.

Notice that these two $\mathbb{Z}$-HS's $S_{K, 1 / n}^{3}$ and $S_{K^{*}, 1 / n}^{3}$ can not be distinguished using the Casson invariant. 
Needless to say, we don't know what kind of geometrical or topological obstruction the invariant $\lambda_{2}$ represents which prevents these two $\mathbb{Z}$-HS's from being homeomorphic.

See [30] for more on this kind of applications of Ohtsuki's invariants.

9. Open questions. Compare with the well developed theory of finite type knot invariants [3, 6, 14, 28, 21, 45, 47], much less have been done in the study of finite type $\mathbb{Z}$-HS invariants. Many questions have been asked in the literature of this subject. We will address a few questions here which are either not asked before or, in the case they are, picked up again because of their significance in our point of view.

Question 1. Is there any other justification for Definition 2.2 besides its analogy to the definition of finite type knot invariants and the fact that it works for the coefficients of the asymptotic expansion of Witten's Chern-Simons path integral at the trivial connection $[41,42]$ ?

One of the original sources of the definition of finite type knot invariants is the study of the topology of the singularities in some functional spaces [47]. See also [45] and [28] for treatments using only elementary topology in 3-dimension. Such a topological interpretation provides us the most natural explanation of the 4-term relation and why it alone suffices essentially to define a "weight system". We don't have such a picture yet for the definition of finite type $\mathbb{Z}$-HS invariants.

Question 2. Does $\mathcal{M} / \mathcal{M}_{k}$ constitute an abelian group under the connected sum? What is the most natural operation which changes a $\mathbb{Z}$-HS to another one preserving the value of any finite type $\mathbb{Z}$-HS invariant up to a fixed order? If we have such an operation on $\mathbb{Z}$-HS's, is there any topological property of $\mathbb{Z}$-HS's which is preserved by this operation?

The first question here is certainly motivated by Gusarov's work [14] and the second by, among others, say, Stanford's work [46]. The operation on knots defined by Stanford is to insert a pure braid commutator somewhere in a given knot. Notice that Gusarov has reported (Oberwolfach, September 1995) that Stanford's operation actually generates all knot with the same value for any finite type invariant up to a fixed order. Finally, the answer to the last question above seems to be not known even for Stanford's operation on knots. Because of its significance, it seems to be worthwhile to repeat it again: Is there any topological property of knots which is preserved by Stanford's operation?

Question 3. Does $\lambda_{2} / 3$ count algebraically any geometrical or topological objects related with the $\mathbb{Z}$-HS in question?

This is certainly motivated by the fact that $\lambda_{1} / 6$ is the Casson invariant, which counts algebraically the number of conjugacy classes of irreducible representations into $S U(2)$ of the fundamental group of the $\mathbb{Z}-\mathrm{HS}$ in question [1]. Probably, the only way to answer this question is to define some counting invariants for $\mathbb{Z}$-HS's first and then identify it with $\lambda_{2}$ using the surgery formula (5.1).

Notice that the geometric definition of the Casson invariant together with its surgery formula leads to a criterion for detecting knots with property P. An answer to Question 3 will provide us another such criterion. 
QUESTION 4. Is it true that if $\lambda$ is a $\mathbb{Z}$-HS invariant of order $\leq k$, then there is a constant $C$ such that for every ASL $L$ in $S^{3}$ with $\mu$ components, we have

$$
\left|\lambda\left(S_{L}^{3}\right)\right| \leq C \mu^{k} ?
$$

Do Ohtsuki's invariants $\lambda_{n}$ have this property?

A similar estimate for finite type knot invariants is conjectured in [29] and proved in [4]. The question to $\lambda_{n}$, with some additional control over the constants $C$ involved, is probably related with the convergence problem of the formal power series

$$
\sum_{n=0}^{\infty} \lambda_{n}(t-1)^{n} .
$$

See [22] for examples of 3-manifolds for which this formal power series does converge.

10. New developments. There have been some major new developments since the submission of this article in October, 1995. In what follows, for the reader's convenience, we give brief summaries of these new works.

10.1. In an important work of Le, Murakami and Ohtsuki [25], a universal 3-manifold invariant $\Omega(M)$ is constructed, which takes values in the completed, graded commutative and cocommutative Hopf algebra of trivalent graphs subject to the $A S$ and $I H X$ relations. To start with, let us denote by $\mathcal{A}(X)$ the graded vector space generated by graphs with univalent and trivalent vertices supported on $X$ where

- $X$ can be the empty space $\emptyset$, a set of $m$ ordered points (which will be denoted simply by $m$ ), and the disjoint union of oriented circles $\coprod S^{1}$;

- all univalent vertices are on $X$;

- when $X$ is a set of $m$ ordered points, points in $X$ are all we have for univalent vertices, and when $X=\coprod S^{1}$, there should be no separate subgraphs with only trivalent vertices;

- a cyclic ordering of the edges at every trivalent vertex is given;

- the grading is given by a half of the number of vertices;

- linear combination of graphs are subject to the $A S$ and $I H X$ relations, and the $S T U$ relations if $X=\coprod S^{1}$ (see [3]).

Denote by $\hat{\mathcal{A}}(X)$ the completion of $\mathcal{A}(X)$.

With these said, the construction in [25] proceeds as follows:

STEP 1. Construct elements $T_{m}^{n} \in \mathcal{A}^{(m-n)}(m)$, which are invariant under cyclic permutations of the $m$ supporting points and characterized essentially by a certain kind of "crossing change formulae".

STEP 2. For a framed link $L$, renormalize the universal invariant $\hat{Z}(L)$ coming from Kontsevich's construction [23] to get an invariant $\breve{Z}(L) \in \hat{\mathcal{A}}\left(\coprod S^{1}\right)$, which is a "group-like element".

STEP 3. Define a linear map

$$
\iota_{n}: \mathcal{A}^{(k)}\left(\coprod S^{1}\right) \longrightarrow \mathcal{A}^{(k-n)}(\emptyset)
$$


by "installing" $T_{2 k}^{n}$ onto each circle so that univalent vertices on both sides match in cyclic order, and then drop off the circles. This extends to a linear map

$$
\iota_{n}: \hat{\mathcal{A}}\left(\coprod S^{1}\right) \longrightarrow \hat{\mathcal{A}}(\emptyset)
$$

STEP 4. $\iota_{n}(\breve{Z}(L)) \in \hat{\mathcal{A}}(\emptyset)$, modulo $\hat{\mathcal{A}}^{(>n)}(\emptyset)$, turns out to be independent of the orientation of $L$ and invariant under the type II Kirby move on the framed link $L$. So, an appropriate normalization of $\iota_{n}(\breve{Z}(L)) \in \hat{\mathcal{A}}(\emptyset)$ modulo $\hat{\mathcal{A}}^{(>n)}(\emptyset)$ gives us an invariant $\Omega_{n}(M)$ of the 3 -manifold $M$ obtained by surgery on $L$.

STEP 5 . The degree $<n$ parts of $\Omega_{n}(M)$ turn out to be determined by the top degree $k$ part of $\Omega_{k}(M)$ denoted by $\Omega_{k}^{(k)}(M)$, for all $k<n$. So, finally, let

$$
\Omega(M)=1+\sum_{n=1}^{\infty} \Omega_{n}^{(n)}(M) \in \hat{\mathcal{A}}(\emptyset) .
$$

It turns out that $\Omega(M)$ is also a "group-like element".

More specifically, we have $\Omega_{n+1}^{(\leq n)}(M)=d \Omega_{n}(M)$, where $d$ equals the cardinality of $H_{1}(M ; \mathbb{Z})$ if the first Betti number of $M$ is 0 and $d=0$ otherwise. So, if $M$ is a $\mathbb{Z}$-HS,

$$
\Omega(M)=1+\sum_{n=1}^{\infty} \Omega_{n}(M) .
$$

When both $M_{1}$ and $M_{2}$ are $\mathbb{Z}$-HS's, we have

$$
\Omega\left(M_{1} \sharp M_{2}\right)=\Omega\left(M_{1}\right) \times \Omega\left(M_{2}\right)
$$

It was proved in [26] that if $M$ is a $\mathbb{Z}$-HS, $\Omega_{1}(M)$ is essentially the Casson invariant.

We remark that this construction of Le, Murakami and Ohtsuki has the similar feature as the construction of $S U(2)$ quantum invariants given by Lickorish [32]. The role played by the elements $T_{m}^{n}$ in the Le-Murakami-Ohtsuki construction is very much like the role played by the so-called Jones-Wenzl projectors in Lickorish's construction. Are they related in a certain way?

10.2. In [24], Le explored further the universal invariant $\Omega(M)$ in the case when $M$ is a $\mathbb{Z}$-HS and made the combinatorial aspect of the theory of finite type invariants of integral homology 3-spheres completely parallel to the theory of finite type invariants of knots. Main results of [24] include (all manifolds below are $\mathbb{Z}$-HS's):

- $\Omega_{n}(M)$ is an invariant of order $\leq 3 n$. This implies, via [11], that the algebra of finite type invariants is isomorphic to the algebra of weight systems, where a weight system is a linear functional on $\mathcal{A}^{(n)}(\emptyset)$.

- An operation on $\mathbb{Z}$-HS's is defined which does not alter the value any invariant of order $\leq n$. This operation is a straightforward generalization of Stanford's operation.

- $\mathcal{M} / \mathcal{M}_{k}$ constitutes an abelian group under the connected sum.

So parts of Question 2 in Section 9 have been answered.

10.3. In [12], the notion of finite type invariants of rational homology 3 -spheres $(\mathbb{Q}$ HS's) is introduced. It is shown that the space of finite type $\mathbb{Q}$-HS invariants is a subspace of finite type $\mathbb{Z}$-HS invariants. In particular, the Casson-Walker invariant [48] of $\mathbb{Q}$-HS's 
is shown to be of order 3 using its surgery formula. In [24], Le also proved that $\Omega_{n}(M)$ is of order $\leq 3 n$ for $\mathbb{Q}$-HS's.

10.4. The recent work of Garoufalidis and Levine [10] provides another perspective to the theory of finite type $\mathbb{Z}$-HS invariants. It relates the stratified vector space $\mathcal{M}$ with the group algebras of some subgroups of the mapping class group completed by powers of their argumentation ideals. The subgroups of the mapping class group are those which are closely related with integral homology 3 -spheres. For example, the Torelli group and the subgroup of the mapping class group generated by Dehn surgeries on bounding curves on surfaces (the main result is stated differently though in these two cases). Recall that the structure of the latter subgroup has been used by S. Morita to recover the Casson invariant $[35,36]$. So, [10] will probably give us some hints as of how to generalize Morita's work to recover Ohtsuki's invariant $\lambda_{2}$ (see Question 3 of Section 9).

\section{References}

[1] S. Akbulut and J. McCarthy, Casson's invariant for oriented homology 3-spheres: An exposition, Math. Notes, vol. 36, Princeton University Press, 1990.

[2] D. Bar-Natan, Perturbative aspects of the Chern-Simons topological quantum field theory, Ph. D. Thesis, Princeton University, 1991. Appeared as Perturbative Chern-Simons theory, Jour. Knot Theory Rami., 14(1995).

[3] D. Bar-Natan, On the Vassiliev knot invariants, Topology, 34(1995).

[4] D. Bar-Natan, Polynomial invariants are polynomial, Math. Res. Letter, 2(1995).

[5] D. Bar-Natan and S. Garoufalidis, On the Melvin-Morton-Rozansky conjecture, preprint, 1994.

[6] J. Birman and X.-S. Lin, Knot polynomials and Vassiliev invariants, Invent. Math., 111(1993)

[7] S. Garoufalidis, On finite type 3-manifold invariants I, to appear in Jour. of Knot Theory Rami.

[8] S. Garoufalidis and J. Levine, On finite type 3-manifold invariants II, preprint, June 1995.

[9] S. Garoufalidis and J. Levine, On finite type 3-manifold invariants IV: comparison of definitions, preprint, September 1995.

[10] S. Garoufalidis and J. Levine, Finite type 3-manifold invariants, the mapping class group and blinks, preprint, March 1996.

[11] S. Garoufalidis and T. Ohtsuki, On finite type 3-manifold invariants III: manifold weight systems, preprint, August 1995.

[12] S. Garoufalidis and T. Ohtsuki, On finite type 3-manifold invariants $V$ : rational homology 3-Spheres, preprint, preprint, November 1995.

[13] M. Greenwood and X.-S. Lin, On Vassiliev knot invariants induced from finite type 3-manifold invariants, preprint, May 1995.

[14] M. Gusarov, On n-equivalence of knots and invariants of finite degree, Adv. in Sov. Math., 18(1994).

[15] N. Habegger, Finite type 3-manifold invariants: a proof of a conjecture of Garoufalidis, preprint, July 1995. 
[16] G. Hardy and E. Wright, An introduction to the theory of numbers, Oxford University Press, Fifth Edition, 1979.

[17] J. Hoste, A formula for Casson's invariant, Tran. AMS, 297(1986).

[18] V. Jones, Hecke algebra representations of braid groups and link polynomials, Ann. of Math., (2)186(1987).

[19] L. Kauffman, The Conway polynomial, Topology, 20(1981).

[20] R. Kirby and P. Melvin, The 3-manifold invariants of Witten and Reshetikhin-Turaev for $\operatorname{sl}(2, \mathbb{C})$, Invent. Math., 105(1991).

[21] M. Kontsevich, Vassiliev's knot invariants, Adv. in Sov. Math., 16(1993).

[22] R. Lawrence, Asymptotic expansion of Witten-Reshetikhin-Turaev invariants for some simple 3-manifolds, to appear in Jour. of Math. Phys.

[23] T. Q. T. Le and J. Murakami, Representations of the category of tangles by Kontsevich's iterated integral, Comm. in Math. Phys., 168(1995).

[24] T. Q. T. Le, An invariant of integral homology 3-spheres which is universal for all finite type invariants, preprint, January 1996.

[25] T. Q. T. Le, J. Murakami and T. Ohtsuki, On a universal invariant of 3-manifolds, preprint, December 1995.

[26] T. Q. T. Le, H. Murakami, J. Murakami and T. Ohtsuki, A three-manifold invariant derived from the universal Vassiliev-Kontsevich invariant, Proc. Japan Acad., Ser. A, 71(1995).

[27] J. Levine, Surgery on links and the $\bar{\mu}$-invariants, Topology, 26(1987).

[28] X.-S. Lin, Finite type link invariants of 3-manifolds, Topology, 33(1994).

[29] X.-S. Lin and Z. Wang, Integral geometry of plane curves and knot invariants, to appear in Jour. of Diff. Geom.

[30] X.-S. Lin and Z. Wang, On Ohtsuki's invariants of integral homology 3-spheres I, preprint, September 1995.

[31] X.-S. Lin and Z. Wang, On Ohtsuki's invariants of integral homology 3-spheres II, in preparation.

[32] W. B. R. Lickorish, Three-manifold invariants and the Temperley-Lieb algebra, Math. Ann., 209(1991).

[33] P. Melvin and H. Morton, The coloured Jones function, Comm. Math. Phys., 169(1995).

[34] J. Milnor and J. Moore, On the structure of Hopf algebras, (2)81(1965).

[35] S. Morita, Casson's invariant for homology 3-spheres and characteristic classes of vector bundles, Topology, 28(1989).

[36] S. Morita, On the structure of Torelli group and the Casson invariant, Topology, 30(1991).

[37] H. Murakami, Quantum SU(2)-invariants dominate Casson's SU(2)-invariant, Math. Proc. Camb. Phil. Soc., 115(1994).

[38] T. Ohtsuki, A polynomial invariant of integral homology 3-spheres, Math. Proc. Camb. Phil. Soc., 117(1995).

[39] T. Ohtsuki, Finite type invariants of integral homology 3-spheres, to appear in Jour. of Knot Theory Rami.

[40] J. Przytycki, Vassiliev-Gusarov skein modules of 3-manifolds and criteria for periodicity of knots, in Low-Dimensional Topology (ed. K. Johannson), International Press Co., 1994.

[41] L. Rozansky, The trivial connection contributions to Witten's invariants of rational homology spheres, preprint, 1995. 
[42] L. Rozansky, Witten's invariants of rational homology spheres at prime values of $K$ and trivial connection contribution, preprint, 1995.

[43] L. Rozansky, On p-adic convergence of perturbative invariants of some rational homology spheres, preprint, 1996.

[44] N. Y. Reshetikhin and V. G. Turaev, Invariants of 3-manifolds via link polynomials and quantum groups, Invent. Math., 103(1991).

[45] T. Stanford, Finite type invariants of knots, links, and graphs, to appear in Topology.

[46] T. Stanford, Braid commutators and Vassiliev invariants, to appear in Paci. Jour. of Math.

[47] V. A. Vassiliev, Cohomology of knot spaces, in Theory of Singularities and its Applications (ed. V. Arnold), AMS, 1990.

[48] K. Walker, An extension of Casson's invariant, Ann. Math. Studies, vol. 121, Princeton Univ. Press, 1991. 